\title{
Trickle-bed CFD studies in the catalytic wet oxidation of phenolic acids
}

\author{
Rodrigo J.G. Lopes, Rosa M. Quinta-Ferreira* \\ Department of Chemical Engineering, University of Coimbra, Rua Sílvio Lima, Polo II, Pinhal de Marrocos, 3030-790 Coimbra, Portugal
}

Received 20 April 2007; received in revised form 11 July 2007; accepted 13 August 2007

Available online 20 September 2007

\begin{abstract}
An Euler-Euler computational fluid model was developed successfully for the hydrodynamic prediction of a trickle-bed reactor (TBR) designed for advanced wastewater treatment facilities. Catalytic wet air oxidation of phenolic acids was simulated in a TBR by means of computational fluid dynamic (CFD) in the temperature range $170-200^{\circ} \mathrm{C}$ and pressures $10-30$ bar. The hydrodynamic model validation was accomplished through the comparison of simulated pressure drop and liquid holdup with experimental data from the literature. In a broad range of gas and liquid flows studied $\left(G=0.10-0.70\right.$ and $\left.L=0.5-5 \mathrm{~kg} / \mathrm{m}^{2} \mathrm{~s}\right)$ at different operation conditions, CFD demonstrated the considerable effect of operating pressure in pressure drop, whereas a minor influence was detected for the liquid holdup. CFD runs were then performed for the catalytic wet air oxidation of aqueous phenolic acids solution. The reactor behaviour was analysed by means of total organic carbon profiles which reflected the influence of temperature, pressure, gas-liquid flows and initial pollutant concentration.
\end{abstract}

(C) 2007 Published by Elsevier Ltd.

Keywords: Computational fluid dynamics; Catalytic wet oxidation; Multiphase flow; Pressure drop; Liquid holdup

\section{Introduction}

The growing public awareness and the increased demand for industrials to meet human requirements have created global problems involving overexploitation of available natural resources leading to pollution of the land, air and water environments. As a consequence, the stringent regulations established by the various governmental authorities are forcing industry to treat effluents to the required compliance level before discharge into the surroundings. Phenols and phenolic acids commonly appear in aqueous final streams arriving from different sources such as food and agro industry as well as pharmaceutical, petrochemical and chemical companies. Unless the concentration is low enough, phenolic wastewaters are poorly biodegradable because of their bactericidal properties (Paraskeva and Diamadopoulos, 2006). Therefore, phenolic compounds must be specifically destroyed before discarding the effluent for subsequent treatment in conventional sewage plants. Among the advanced oxidation processes, catalytic wet air oxidation (CWAO) has been shown to be an effective

\footnotetext{
* Corresponding author. Tel.: +351 239798723; fax: +351 239798703.

E-mail addresses: rodrigo@eq.uc.pt (R.J.G. Lopes), rosaqf@eq.uc.pt (R.M. Quinta-Ferreira).
}

technique for eliminating organic compounds, such as phenolic and other aromatic products (Bhargava et al., 2006). In this ambit, catalyst screening studies have been performed successfully in our group with a model solution of several phenolic acids including syringic, vanillic, 3,4,5-trimethoxybenzoic, veratric, protocatechuic and 4-hidroxybenzoic acids in which total organic carbon (TOC) was oxidised in batch wise slurry reactor (Lopes et al., 2007a). In particular, the $\mathrm{Mn}-\mathrm{Ce}-\mathrm{O}$ catalyst revealed good stability in terms of leaching and carbon adsorption leading to the complete TOC removal so that catalyst deactivation phenomena were not addressed in the present study.

However, at industrial level, three-phase reactors are required for the continuous wastewater treatment operating in trickle flow regime at trickle-bed reactors (TBR). In order to achieve industrial feasibility, four main aspects of CWO in continuous mode have to be considered namely chemistry of CWO, reactor geometry, catalyst stability and scale-up (Sie and Krishna, 1998). If the first parameters have been reviewed in the literature, scheduling and scale-up studies leading to their applicability to industrial processes on CWO reaction engineering have not been fulfilled. Therefore, the present paper intends to examine the behavior of a TBR by means of computational fluid dynamic (CFD) codes. 
The use of TBRs is common in industrial multiphase catalytic processes and spans a broad range of applications from the manufacture of value-added products to the conversion of undesired chemicals into harmless and bio-compatible species. Despite nearly 50 years of worldwide research efforts, a satisfactory approach to TBRs is still out of grasp. Even though fluid dynamics continue to be among the most intensely studied areas, TBR state-of-the-art is far from being complete and yet no universal approach has emerged as a panacea to predict conclusively TBR key fluid dynamic parameters (Al-Dahhan et al., 1997; Carbonell, 2000; Gianetto and Specchia, 1992; Goto and Smith, 1975; Sáez and Carbonell, 1985). This is partly ascribable to the diverse entangling gas-liquid patterns met in a TBR which make such parameters depend in a complex manner on the fluid properties and throughputs, interfacial interactions and bed geometry. In these reactors, gas and liquid phase percolate co-currently downward through a fixed bed of catalyst particles. Generally, three-phase fixed bed reactors can operate in hydrodynamically different regimes whose boundaries depend on gas and liquid superficial velocities, catalyst bed and fluid properties (Al-Dahhan et al., 1997; Attou and Ferschneider, 1999; Holub et al., 1993; van der Merwe and Nicol, 2005).

In practice, many of the chemical reactors used in industry are truly multiphase and must be described in the context of CFD by multiple momentum equations (Jiang et al., 2002). Direct numerical simulation of the transport equations for all phases with fully resolved interfaces between phases is possible for only the simplest systems. Such a detailed model could not be used to predict a large chemical reactor such as a TBR and other multiphase reacting flows (Gunjal et al., 2005). As a middle-term approach, we evaluated in a previous work the TBR behaviour for the catalytic wet oxidation of a vanillic acid solution by using the available methods in commercial CFD codes where the fluid is modelled by the volume or mass fractions for each phase (Lopes et al., 2007b) much in the same way used to describe micromixing in single-phase flows.

\section{Numerical model and governing flow equations}

In this work, TBR is modelled by means of a multifluid CFD Euler-Euler two-fluid model implemented in commercial software FLUENT 6.1. In a first step, the hydrodynamic behaviour for the gas-liquid concurrent downflow is analysed in order to validate with results taken from the open literature. Afterwards, the influence of operation conditions on the catalytic wet oxidation in continuous mode is evaluated. At the subgrid scale, the two phases $(G / L)$ are described by the corresponding volume fractions. The multifluid CFD model at its most basic level consists of mass and momentum balances for each phase. In the case of multiphase and incompressible flow, the pressure constrains the velocity field to ensure that the sum of the phase volume fractions equals unity. To reduce the computational requirements and numerical efforts, the mesh domain was previously optimized in terms of cell number.

FLUENT uses phase-weighted averaging for turbulent multiphase flow, and then no additional turbulent dispersion term is introduced into the continuity equation. The mass conservation equation for each phase is

$\frac{\partial}{\partial t}\left(\rho_{i} \alpha_{i}\right)+\nabla \cdot\left(\alpha_{i} \rho_{i} \vec{u}_{i}\right)=0$,

where $\rho_{i}, \alpha_{i}$ and $\vec{u}_{i}$ represent the density, volume fraction and mean velocity, respectively, of phase $i(L$ or $G)$. As referred, the liquid phase $L$ and the gas phase $G$ are assumed to share space in proportion to their volume such that their volume fractions sums to unity in the cells domain

$\alpha_{L}+\alpha_{G}=1$.

The momentum conservation equation for the phase $i$ after averaging is

$$
\begin{aligned}
\frac{\partial}{\partial t}\left(\rho_{i} \alpha_{i} \vec{u}_{i}\right)+\nabla \cdot\left(\rho_{i} \alpha_{i} \vec{u}_{i} \vec{u}_{i}\right)= & -\alpha_{i} \nabla p+\nabla \cdot \overline{\bar{\tau}}_{\text {eff }}+\vec{R}_{i}+\vec{F}_{i} \\
& +\rho_{i} \alpha_{i} \vec{g} .
\end{aligned}
$$

$p$ is a pressure shared by the two phases and $\vec{R}_{i}$ represents the interphase momentum exchange terms. The Reynolds stress tensor $\overline{\bar{\tau}}_{\text {eff }}$ is related to the mean velocity gradients using a Boussinesq hypothesis:

$$
\begin{aligned}
\overline{\bar{\tau}}_{\text {eff }}= & \alpha_{i}\left(\mu_{\text {lam }, i}+\mu_{t, i}\right)\left(\nabla \vec{u}_{i}+\nabla \vec{u}_{i}^{T}\right) \\
& -\frac{2}{3} \alpha_{i}\left(\rho_{i} k_{i}+\left(\mu_{\mathrm{lam}, i}+\mu_{t, i}\right) \nabla \cdot \vec{u}_{i}\right) \overline{\bar{I}} .
\end{aligned}
$$

The most important interphase force is the turbulent drag force resulting from the mean relative velocity between the two phases and an additional contribution resulting from turbulent fluctuations in the volume fraction due to averaging of momentum equations (FLUENT 6.1,2005). $\vec{R}_{i}$ is reduced only to the drag force proportional to the mean velocity difference, given by the following form where $K$ is the liquid-gas exchange coefficient as described in the following equation:

$\vec{R}_{L}=-\vec{R}_{G}=K\left(\vec{u}_{G}-\vec{u}_{L}\right)$.

Mainly due to the intrinsic nature of multiphase reactors, the incomplete understanding of the physics plus the highly coupled and nonlinear nature of the equations, the complete solution is largely dependent in the mechanistic principles and advanced numerical analysis as well as satisfactory computational resources. Therefore, closures equations for fluid/fluid and fluid/particle interactions integrated in the overall momentum balance equation is often an approximation based in theoretical assumptions. The left-hand side of Eq. (3) represents the rate of change of momentum for the $i$ th phase, whereas the right-hand side represents pressure forces, gravitational acceleration, average shear stresses and interphase momentum exchange. The pressure drop in the packed bed is usually correlated using the Ergun equation or its variants (Al-Dahhan et al., 1997; Holub et al., 1993; Sáez and Carbonell, 1985). Interphase coupling terms may therefore be formulated based on similar equations. The presence of liquid flow, however, leads to additional interphase exchanges, which need to be formulated correctly. We have used the model of Attou and Ferschneider, 1999, which includes gas-liquid interaction forces and it was 
developed for the regime in which liquid flows in the form of film. The interphase coupling terms are expressed in terms of interstitial velocities and phase volume fractions for gas-liquid, gas-solid and liquid-solid momentum exchange forms:

$$
\begin{aligned}
F_{G L}= & \varepsilon_{G}\left(\frac{E_{1} \mu_{G}\left(1-\varepsilon_{G}\right)^{2}}{\varepsilon_{G}^{2} d_{p}^{2}}\left[\frac{\varepsilon_{S}}{1-\varepsilon_{G}}\right]^{2 / 3}\right. \\
& \left.+\frac{E_{2} \rho_{G}\left(u_{G}-u_{L}\right)\left(1-\varepsilon_{G}\right)}{\varepsilon_{G} d_{p}}\left[\frac{\varepsilon_{S}}{1-\varepsilon_{G}}\right]^{1 / 3}\right), \\
F_{G S}= & \varepsilon_{G}\left(\frac{E_{1} \mu_{G}\left(1-\varepsilon_{G}\right)^{2}}{\varepsilon_{G}^{2} d_{p}^{2}}\left[\frac{\varepsilon_{S}}{1-\varepsilon_{G}}\right]^{2 / 3}\right. \\
& \left.+\frac{E_{2} \rho_{G} u_{G}\left(1-\varepsilon_{G}\right)}{\varepsilon_{G} d_{p}}\left[\frac{\varepsilon_{S}}{1-\varepsilon_{G}}\right]^{1 / 3}\right), \\
F_{L S}= & \varepsilon_{L}\left(\frac{E_{1} \mu_{L} \varepsilon_{S}^{2}}{\varepsilon_{L}^{2} d_{p}^{2}}+\frac{E_{2} \rho_{L} u_{G} \varepsilon_{S}}{\varepsilon_{L} d_{p}}\right) .
\end{aligned}
$$

Gas-liquid, liquid-solid and gas-solid mass transfer is modelled by the Stefan-Maxwell equations for multicomponent transfer. This formulation for interphase mass and energy transport need to be modeled for unsteady state operation due to the fact that assumptions for conventional isothermal, steady state dilute solution transport of single species are not justified in the unsteady state operation case (FLUENT 6.1, 2005). The solution of the Stefan-Maxwell equations involves the formulation of the transport and equilibrium relations at each interface, which are then solved for the required fluxes and compositions (Taylor and Krishna, 1993). Trickle flow is the operating flow regime studied and typically characterized by lower values of Reynolds number, i.e., according to the literature gas-liquid interaction is low enough so capillary pressure force can be neglected in most cases. This means that we can assume same pressure for both phases at any point in time and space. In fact, the contribution of the turbulent stress terms to overall momentum balance equation is not significant in laminar flow (FLUENT 6.1, 2005) so that the reason of accounting for $k-\varepsilon$ turbulence model is primarily based on the fact the wall functions in other models would have limitations in predicting the heat transfer of convective flow because the flow and temperature boundary layer would not be properly simulated, even if a fine boundary layer grid is used. For this reason, when using a $k-\varepsilon$ turbulence model a rather coarse grid should be carefully used at the boundary layer. For example, using the SST turbulence model the flow and temperature distribution in the boundary layer can be simulated but only if the boundary layer is resolved by a fine grid. Therefore, the standard $k-\varepsilon$ turbulence model is not used in this paper to simulate turbulence because its overall effect in momentum equation is negligible, but mainly to account indirectly for heat transport phenomena. The turbulent liquid viscosity $\mu_{t, L}$ is

$\mu_{t, L}=\rho_{L} C_{\mu} \frac{k_{L}^{2}}{\varepsilon_{L}}$ and is obtained from the prediction of the transport equations for the $k_{L}$, kinetic energy, and $\varepsilon_{L}$, dissipation energy in the following equations, respectively

$$
\begin{aligned}
& \frac{\partial}{\partial t}\left(\rho_{L} \alpha_{L} k_{L}\right)+\nabla \cdot\left(\rho_{L} \alpha_{L} \vec{u}_{L} k_{L}\right) \\
& =\nabla \cdot\left(\alpha_{L} \frac{\mu_{t, L}}{\sigma_{k}} \nabla k_{L}\right)+\alpha_{L} G_{k, L}-\alpha_{L} \rho_{L} \varepsilon_{L} \\
& \quad+\alpha_{L} \rho_{L} \Pi_{k L} \\
& \frac{\partial}{\partial t}\left(\rho_{L} \alpha_{L} \varepsilon_{L}\right)+\nabla \cdot\left(\rho_{L} \alpha_{L} \vec{u}_{L} \varepsilon_{L}\right) \\
& \quad=\nabla \cdot\left(\alpha_{L} \frac{\mu_{t, L}}{\sigma_{\varepsilon}} \nabla \varepsilon_{L}\right)+\alpha_{L} \frac{\varepsilon_{L}}{k_{L}} \times\left(C_{1 \varepsilon} G_{k, L}+C_{2 \varepsilon} \rho_{L} \varepsilon_{L}\right) \\
& \quad+\alpha_{L} \rho_{L} \Pi_{\varepsilon L} .
\end{aligned}
$$

$G_{k L}$ is the rate of production of turbulent kinetic energy. $C_{\mu}$, $C_{1 \varepsilon}, C_{2 \varepsilon}, C_{3 \varepsilon}, \sigma_{k}$ and $\sigma_{\varepsilon}$ are parameters of the standard $k-\varepsilon$ model with the following values: $0.09,1.44,1.92,1.2,1.0$ and 1.3. The turbulent quantities for the kinetic energy and turbulent viscosity of the gas were modelled using the primary phase turbulent quantities.

The conservation of energy in Eulerian multiphase applications is described by a separate enthalpy equation for each phase

$$
\begin{aligned}
& \frac{\partial}{\partial t}\left(\alpha_{q} \rho_{q} h_{q}\right)+\nabla \cdot\left(\alpha_{q} \rho_{q} \vec{u}_{q} h_{q}\right)=-\alpha_{q} \frac{\partial p_{q}}{\partial t}+\overline{\bar{\tau}}_{q}: \nabla \vec{u}_{q}-\nabla \cdot \vec{q}_{q} \\
& \quad+S_{q}+\sum_{p=1}^{n}\left(\vec{Q}_{p q}+\dot{m}_{p q} h_{p q}-\dot{m}_{q p} h_{q p}\right),
\end{aligned}
$$

where $h_{q}$ is the specific enthalpy of the $q$ th phase, $\vec{q}_{q}$ is the heat flux, $S_{q}$ is a source term that includes sources of enthalpy (e.g., due to chemical reaction or radiation), $Q_{p q}$ is the intensity of heat exchange between the $p$ th and $q$ th phases, and $h_{p q}$ is the interphase enthalpy (e.g., the enthalpy of the vapour at the temperature of the droplets, in the case of evaporation). The heat exchange between phases must comply with the local balance conditions $Q_{p q}=-Q_{q p}$ and $Q_{q q}=0$.

\section{Results and discussion}

\subsection{TBR specifications and numerical techniques}

The solution domain for the experimental system investigated in this work is shown in Fig. 1. It consists of a cylindrical vessel with $5 \mathrm{~cm}$ internal diameter and $1 \mathrm{~m}$ bed height packed with catalyst spherical particles $2 \mathrm{~mm}$ diameter. Numerical simulations are compared to experimental data in order to validate the predicted hydrodynamic parameters pressure drop and liquid holdup. The simulated operating conditions simulated were $10-30$ bar pressure and temperatures from 290 to $500 \mathrm{~K}$. Gas and liquid mass flow rates were in the range $0.10-0.70$ and $0.05-15 \mathrm{~kg} / \mathrm{m}^{2} \mathrm{~s}$, respectively.

The geometry of the top distributor is not included in the present model and therefore its influence on the flow field is ignored. The FLUENT preprocessor GAMBIT 2 (2005) was 


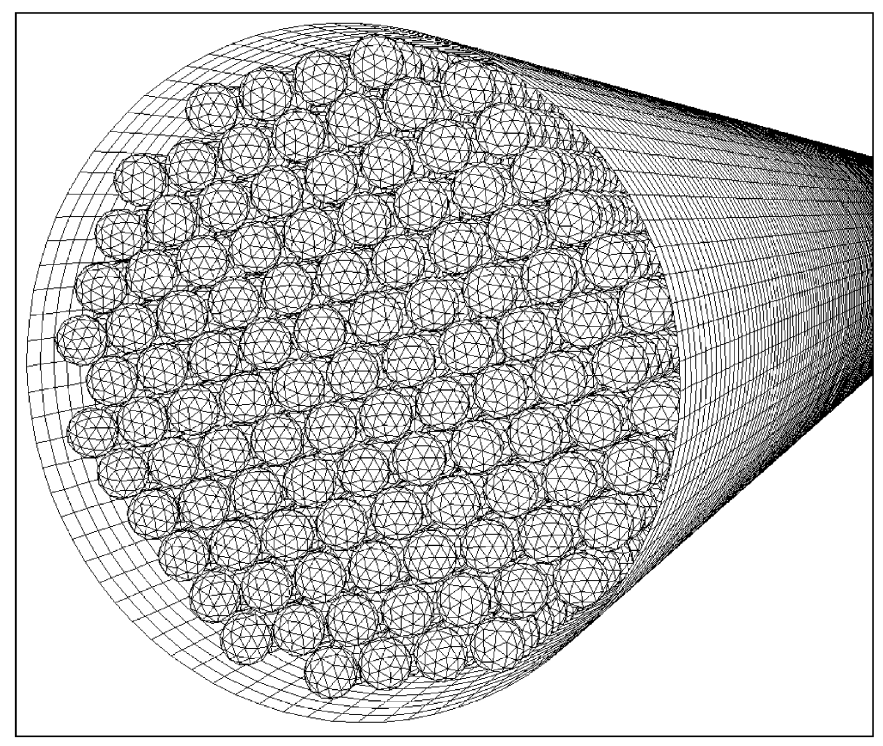

Fig. 1. Computational mesh domain of TBR at the entrance.

used as a geometry and mesh generator. The mesh of the packing bed was designed excluding surface roughness. The multigrid computational domain adopted in the TBR is characterized by tetrahedral cells around and over the catalyst particles and hexahedral elsewhere because this strategy takes advantage of the hierarchical nature of the grids, incorporating an efficient technique to generate the coarser grids in the mesh preprocessor GAMBIT 2, 2005. The governing equations are solved with a time step based on the flow speed within that particular mesh that can be useful to save CPU time by requiring coarse meshes to be updated only when necessary and implemented in commercial software FLUENT 6.1. Closures equations are solved using user-defined routines. For that reason and aiming to achieve an higher numerical definition, the compromise is between the application of coarse meshes that are best designed in regions where temporal and spatial gradients of key quantities are relatively small and the preliminary numerical results that were carried out to identify the number of computational cells appropriate to obtain qualitative and quantitatively grid independent results. Fig. 1 shows the essential features of the 800.000 tetrahedral cells representing the catalytic bed. The quality of meshes was analysed using the skewness criteria based on the difference between the cell size and the optimal size which is the equilateral volume. All the cells skewness are below 0.60 which indicates that the mesh is acceptable (GAMBIT 2, 2005). The system domain is discretized by an unstructured finite volume method, obtained using the solver FLUENT 6.1 (2005) in order to convert the governing equations like continuity and momentum equations to algebraic equations that can be solved numerically. The reactor wall and catalyst surfaces are treated as nonslip boundaries with standard wall functions. The gas flow rate at the distributor is defined via inlet-velocity-type boundary condition with gas volume fraction charging according to the specifications made in the simulations. The use of under-relaxation factors represents a good compromise between physical accuracy and reasonable computational effort. The flow model is based on solving Navier-Stokes equations for the Eulerian-Eulerian multiphase model along with muliphase $k-\varepsilon$ turbulent model. The governing differential equations aree solved using iterative solution to the discrete form of the mathematical model using a SIMPLE algorithm for pressure-velocity coupling with first order implicit formulation for unsteady integration and first order upwind scheme discretization for spatial derivatives. It should be pointed out that higher integration schemes can in general give better results at the expense of more computational power. However, in order to assess the usefulness of an Eulerian approach simulating a trickle flow with Newtonian fluids, the effect of discretization schemes on numerical solutions for the convection term in the constitutive equation is more adequate for viscoelastic fluid flows, according and commonly used in the literature. For this purpose, different discretization schemes were not considered in our case study given that the first-order upwind difference scheme and artificial diffusion scheme show most stable and smooth solutions even for highly extensional flows. The gas and liquid are described as interpenetrating continua and equations for conservation of mass and momentum are solved for each phase. To avoid numerical difficulties, the transient calculations were made for two-phase flow starting with different time steps. The converged solution is assumed when the scaled residuals of all variables were smaller than $10^{-3}$. Preliminary numerical simulations were carried out to identify the computational cells that are adequate to obtain grid independent results so that these numerical simulations showed that the predicted values of overall pressure drop and liquid holdup become insensitive to further grid refinement either when increasing the number of grid cells or using higher order discretization scheme that caused less than $5 \%$ change in pressure drop and less than $1 \%$ change in liquid volume fraction.

\subsection{Hydrodynamic studies}

As the performance of the TBR is affected by the fluid dynamic parameters, the hydrodynamic studies are discussed in terms of liquid holdup and pressure drop at different operating pressures in the range 10-30 bar. In order to validate the computational model, the runs were carried out for the vectorial field of liquid and gas velocity and for liquid hold up and pressure drop using spherical catalysts with $2 \mathrm{~mm}$ diameter. The CFD flow maps indicate that for gas (Fig. 2) and liquid (Fig. 3), the velocity is higher at points where the flow is processed downward in axial direction. In accordance to these results, the maximum gas velocity is about $0.5 \mathrm{~cm} / \mathrm{s}$ (Fig. 2) while the liquid velocity is about $0.005 \mathrm{~cm} / \mathrm{s}$ (Fig. 3) which is in the range of well accepted trickle flow maps reviewed elsewhere (Al-Dahhan et al., 1997; Wammes and Westerterp, 1990). Simulated CFD liquid holdup and pressure drop are represented in Figs. 4 and 5 by lines as a function of liquid mass flux water when the reactor operates with air as the gas phase at different pressure values. The experimental data plotted in Figs. 4 and 5 were available from the work developed by Nemec and Levec (2005) in where it was described in detail the 


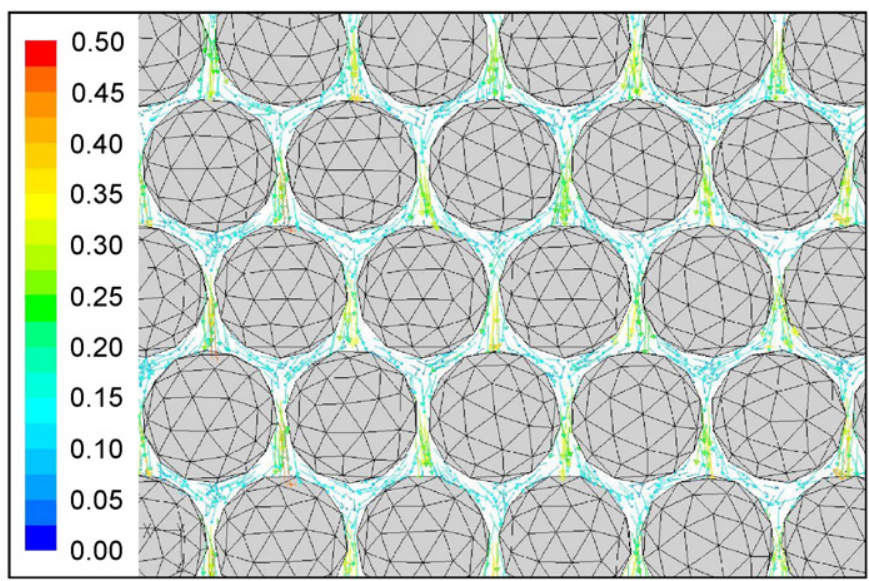

Fig. 2. Gas velocity $(\mathrm{cm} / \mathrm{s})$ map at axisymmetric plane.

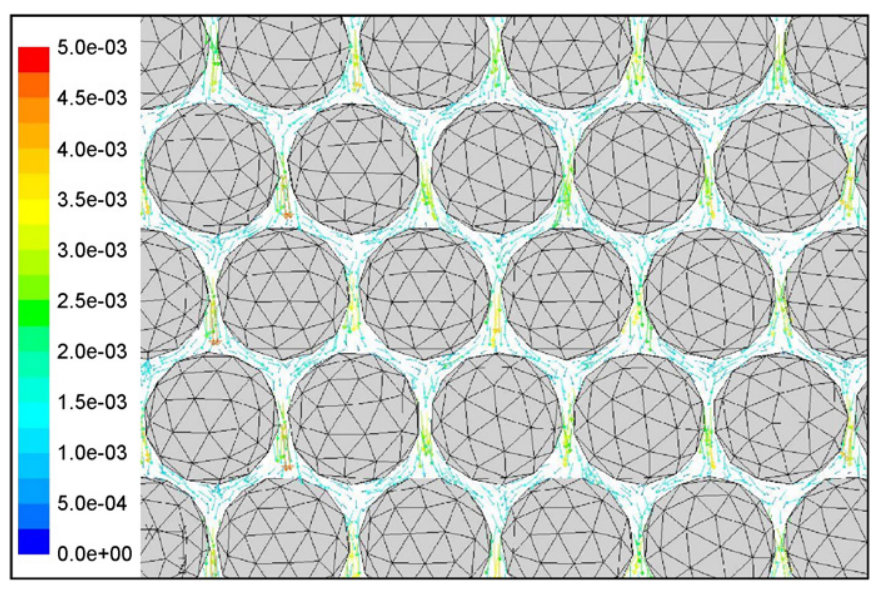

Fig. 3. Liquid velocity $(\mathrm{cm} / \mathrm{s})$ map at axisymmetric plane.

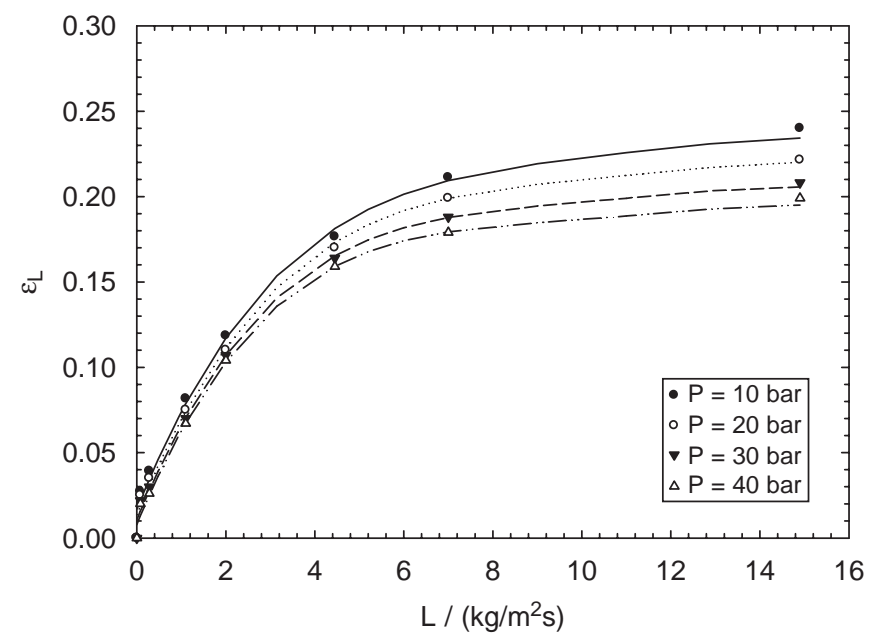

Fig. 4. Liquid holdup as a function of liquid mass flux at constant pressure values $\left(G=0.5 \mathrm{~kg} / \mathrm{m}^{2} \mathrm{~s}\right)$.

experimental setup. In that work, liquid holdup was measured by a gravimetric method that consists in weighing the column in two different ways to have good reproducibility. After the bed was extensively prewetted, the reactor with dimensions similar

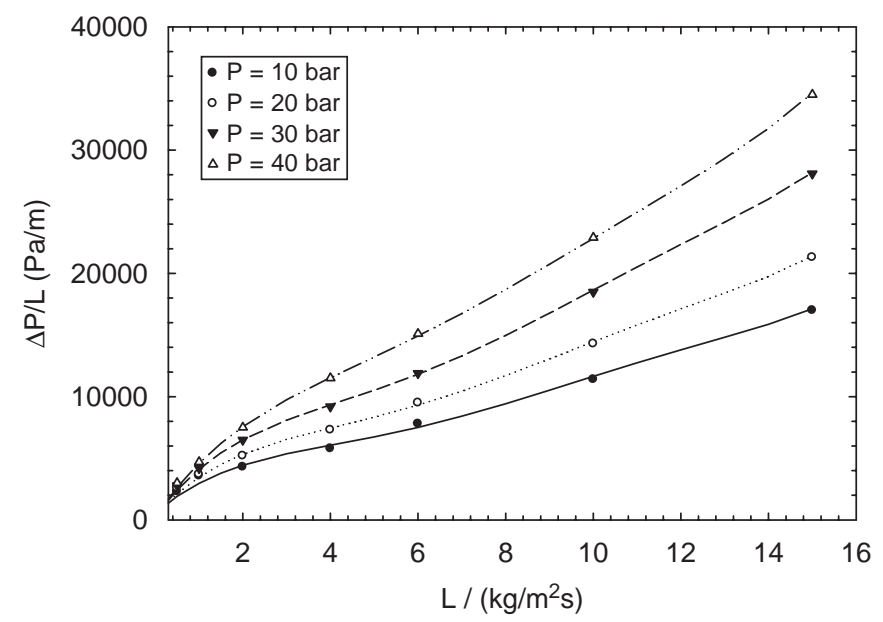

Fig. 5. Pressure drop as a function of liquid mass flux at constant pressure values $\left(G=0.5 \mathrm{~kg} / \mathrm{m}^{2} \mathrm{~s}\right)$.

to the ones described previously was operated first in a high interaction regime and then reduced to the desired level at which the pressure drop and liquid holdup were measured. These predictions are in good agreement with experimental values which enables the validation of our CFD model. In fact, the computational fluid dynamic model validation was carried out first in single-phase pressure drop simulations with only the gas phase flowing downward the bed; afterwards, two-phase flow is simulated to perform the final comparison between predicted hydrodynamic parameters and experimental data. In the whole range of Reynolds numbers for gas phase, pressure drop predictions are within $10 \%$ of error when comparing with the measurements provided by Nemec and Levec (2005). It should be pointed out that the operational region of flow rates $\left(10<R e_{G}<400\right)$ is that of particular interest to TBR and in this ambit Eulerian model fits the pressure drop data as well as liquid holdup quite well within acceptable limits of $10 \%$. Moreover, our validation results are successfully compared against the results of Attou and Ferschneider (1999) for the pressure drop in a parity plot illustrated elsewhere (Lopes et al., 2007b). It should be also emphasized that the fixed-bed modelled in this work had the tube to particle diameter ratio higher than 10 so the available geometry and data taken from literature should not be affected by the reactor column wall. In accordance to Fig. 4, when the liquid mass flux increases, the liquid holdup also increases for L higher than $8 \mathrm{~kg} / \mathrm{m}^{2} \mathrm{~s}$ being the growth rate smaller for the same total pressure value whereas an increase of the total pressure results in a considerable decrease of liquid holdup. The influence of the gas flow determined by a different operating pressure on the liquid holdup is less pronounced at low values of liquid mass fluxes. For example, in case the reactor operates at 40 bar, the liquid holdup is substantially lower compared when it operates at 10 bar. These higher differences at higher $L$ values result from the fact that a further increase of the reactor pressure at a constant gas velocity corresponds to a higher driving force.

The influence of the superficial gas velocity and of the reactor pressure on the liquid holdup can be explained by means of 
the pressure gradient over the reactor, i.e. the drag force at the gas-liquid interface. The pressure gradient depends on the gas velocity and on the gas density; it is together with the gravitational force the driving force for the liquid flow. The liquid holdup is the result of two counteracting forces: the frictional forces at the packing surface and the driving force acting on the liquid phase (Al-Dahhan et al., 1997; Holub et al., 1993; Sáez and Carbonell, 1985). In the case of both liquid and gas flows are present, the total driving force increases due to the pressure gradient; nevertheless, as soon as the liquid mass flux becomes more significant, the liquid holdup remains practically constant at 0.2 as observed by the threshold observed in Fig. 4 and only increases slightly. Comparing Figs. 4 and 5, where the pressure gradient per unit reactor length has been plotted as a function of liquid mass flux, we see that for very low values of pressure drop the liquid holdup are equally small. With the increase of pressure drop due to higher reactor pressures, the total driving force enlarges noticeably and, hence, the liquid holdup growth rate reduces when the liquid mass flux increases (Wammes and Westerterp, 1990). Moreover, the comparison between the hydrodynamic parameters determined at 10 and 40 bar shows that the effect of the reactor pressure has greater influence on the pressure drop than it has on the liquid holdup as expected.

\subsection{Effect of operating conditions on TOC conversion}

Some TBR models reported in the literature considered isothermal operation and used either a pseudo-homogeneous or a heterogeneous model with plug-flow for gas and liquid phase while other models accounted for liquid flow non-uniformity by using an axial dispersion model or even a residence time distribution based model. In the present work, our CFD Euler-Euler two-fluid model incorporate the three fundamental balances, continuity, momentum and energy computed by means of phase-weighted averaging for turbulent multiphase flow.

The TBR oxidation behaviour in terms of total organic carbon conversion is developed taking into account the kinetic expressions obtained elsewhere (Lopes et al., 2007a) dealing with the catalytic wet air oxidation of several phenolic acids. The kinetic parameters of catalytic wet air oxidation for the phenolic mixture were calculated by means of generalized kinetic model (GKM), a lumped kinetic model widely used to describe the total organic carbon profiles in CWO reactions. GKM considers three types of compounds: easier degraded reactants (A); intermediates with difficult degradation (B) and desired end products, namely carbon dioxide and water (C). In the oxidation process of the phenolic solutions with $\mathrm{Mn}-\mathrm{Ce}-\mathrm{O}$ $70 / 30$, phenol and acetic acid were formed as intermediate compounds, being totally degraded during the treatment and the overall TOC practically reduced to zero. The activation energy and the pre-exponential factor were calculated by using the Arrhenius plot and the reaction rate constants as a function of temperature were then integrated in the TBR model as well as the reaction enthalpy computed from the difference between the enthalpy of formation of end products $\left(\mathrm{CO}_{2}\right.$ and $\left.\mathrm{H}_{2} \mathrm{O}\right)$ and initial reagents, considering total organic carbon conversion of the six phenolic acids. In the computational model, the liquid

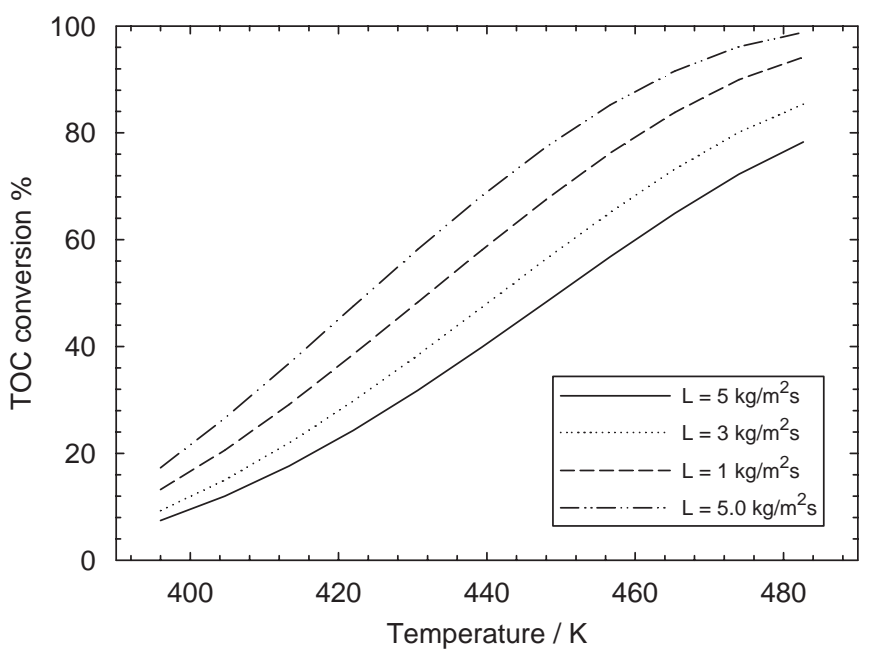

Fig. 6. Bulk temperature $(K)$ at axisymmetric plane without CWAO.

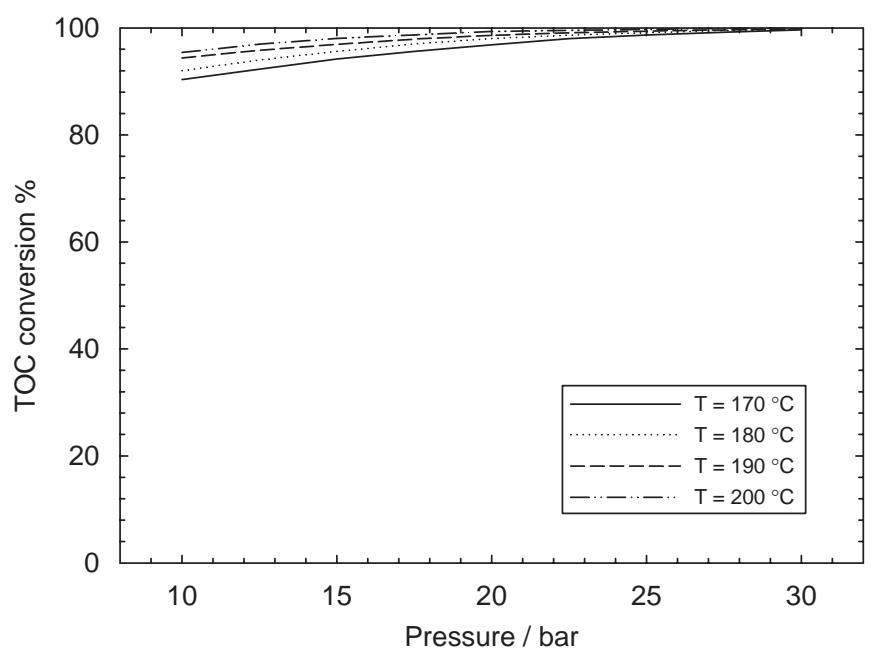

Fig. 7. Bulk temperature $(K)$ at axisymmetric plane with CWAO.

reactants were assumed to be non-volatile and the gas phase is pure at constant partial pressure of the reacting oxygen. The results of our simulations have assumed a uniform flow at the reactor entrance and that the catalyst diameter $(2 \mathrm{~mm})$ has a minor influence in terms of wall flow. In fact, according to the literature recommendations the catalyst geometry has significant effect on the wall flow treatment in FLUENT at catalyst spherical diameters higher than $5 \mathrm{~mm}$. The effectiveness of the TBR for phenolic content conversion was investigated in terms of different reactor heating temperatures, total pressures, gas and liquid mass fluxes and initial concentrations of the pollutant.

The effect of temperature on TOC conversion is shown in Fig. 6 at different liquid mass fluxes. Negligible conversion was obtained for low temperatures and as the temperatures increases, a considerable influence in total organic carbon abatement is observed leading to reduction values higher than $95 \%$ for lower liquid flow rates, i.e. for higher residence times.

Fig. 7 shows that the effect of reactor pressure on conversion is significantly lower than the one observed for the temperature 


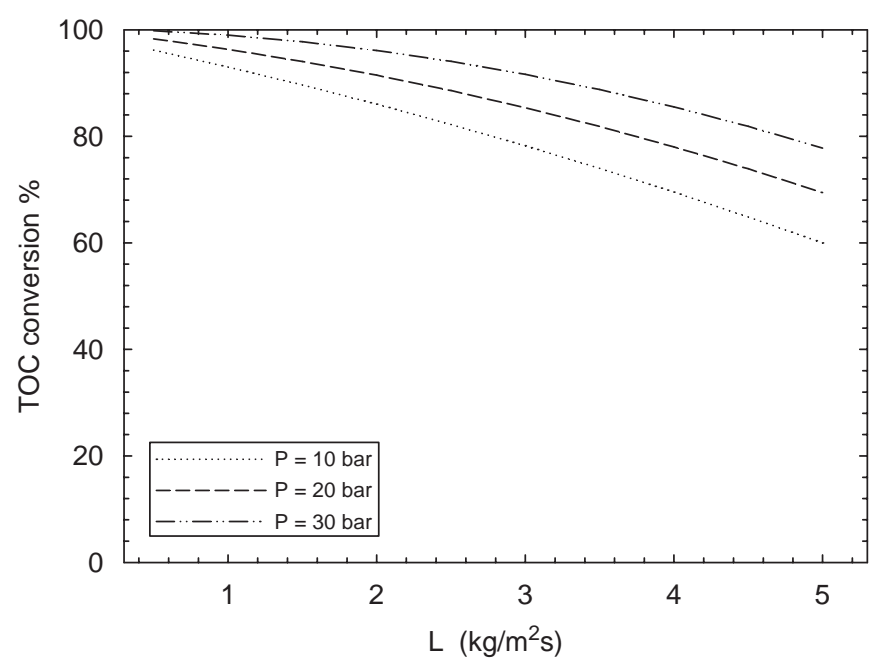

Fig. 8. TOC conversion as a function of mean bed temperature for different liquid mass fluxes $\left(G=0.5 \mathrm{~kg} / \mathrm{m}^{2} \mathrm{~s} ; P=30 \mathrm{bar}\right)$.

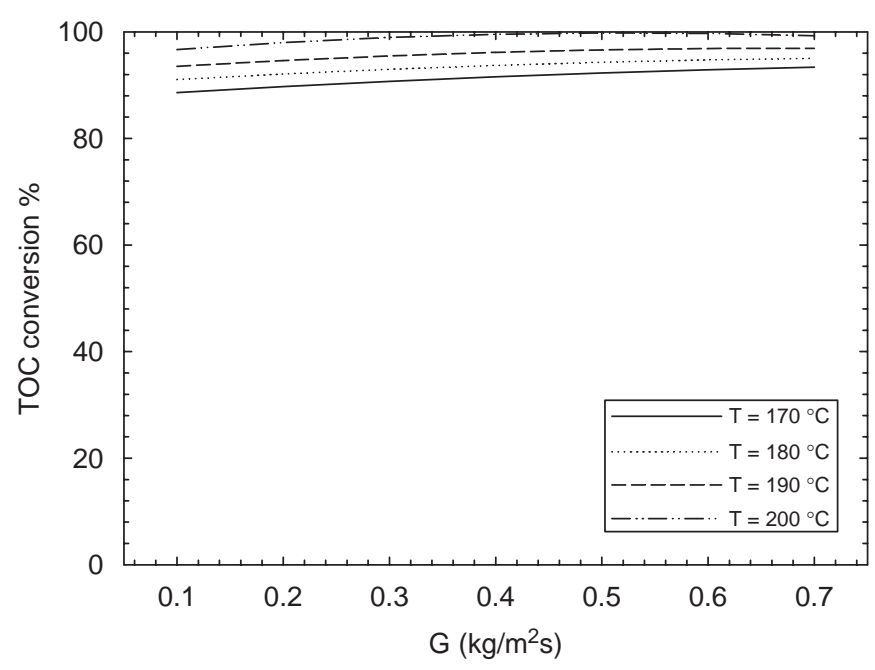

Fig. 9. TOC conversion as a function of operating pressure for different temperatures $\left(L=0.5 \mathrm{~kg} / \mathrm{m}^{2} \mathrm{~s}\right)$.

and it can be seen that increasing pressure from 10 to 30 bar leads to higher TOC conversions. Therefore, for higher pressures, the gas density and its solubility also increases in the liquid which provides additionally that increase in gas pressure provides a lateral push force for the reactants to cover as much surface area as possible.

The effect of liquid mass flux on conversion is shown in Fig. 8 at different pressures while the result of gas mass flux on TOC degradation is represented in Fig. 9. TOC conversion gradually decreased with increasing liquid mass flux since the corresponding lower residence times of the reactant reduce the reaction time of pollutant. Moreover, higher liquid flow rates give greater liquid hold up which decreases the contact of liquid and gas reactants at the catalyst active site by increasing the film thickness. It has also been observed that the gas flow rate has not the same effect in TOC conversion. Fig. 9 shows a plot for conversion against gas mass flux revealing that for

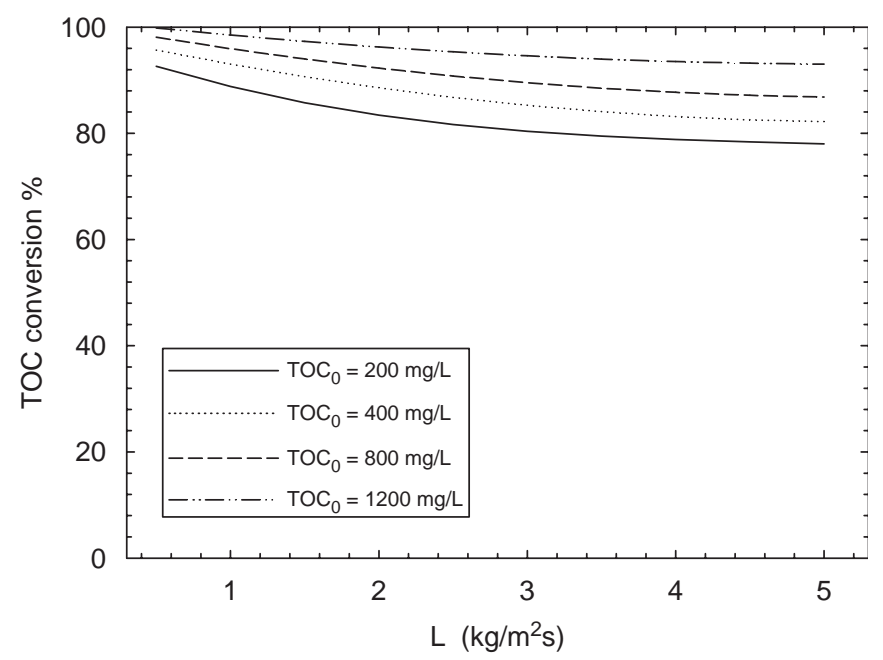

Fig. 10. TOC conversion as a function of liquid mass flux for different pressures $\left(G=0.5 \mathrm{~kg} / \mathrm{m}^{2} \mathrm{~s}\right)$.

higher temperatures the degradation may reach a maximum value, increasing initially with gas flow rate and decreasing afterwards. This fact could be interpreted by the improvement achieved for higher temperatures in the distribution of liquid film over catalyst and hence wetting increases as observed by several authors.

The effect of inlet TOC concentration on CWO is shown in Fig. 10 from 200 to $1200 \mathrm{mg} / \mathrm{L}$ improving TOC degradation. As the solution is highly diluted, any augment in initial TOC content leads to higher oxidation rates and hence better conversions.

\section{Conclusions}

A trickle-bed reactor designed for the catalytic wet air oxidation of phenolic acids was modelled by means of computational fluid dynamics. The model consists in a Euler-Euler treatment for the fluid phases coupled with the energy equation. The numerical simulations are compared against experimental data to validate the predicted hydrodynamic parameters pressure drop and liquid holdup. Operating conditions were simulated with 10-30 bar of reactor pressure while gas and liquid mass flow rate were in the range $0.10-0.70$ and $0.05-15 \mathrm{~kg} / \mathrm{m}^{2} \mathrm{~s}$, respectively.

The hydrodynamic studies pointed out that the liquid holdup increases as the liquid mass flux increases and decreases for higher operating pressure values. At low values of pressure drop the liquid holdup is small but with an increase value of pressure drop due to an increase of the reactor pressure, the liquid holdup growth rate reduces when the liquid mass flux increases. The influence of operating pressure on liquid holdup is less pronounced than it has on pressure drop.

Afterwards, the effect of operation conditions on TOC conversion is discriminated in terms of temperature, pressure, gas-liquid flow rate and initial pollutant concentration. TOC conversion depends heavily on the temperature bed while the operating pressure has minor influence in final conversion. 
When the liquid flow rate is decreased, the residence time increase and the conversion is higher but increasing the gas flow rate it was achieved an optimum value where the TOC conversion is maximum. Moreover, higher values of inlet pollutant concentration led also to higher conversions.

\section{Notation}

$\begin{array}{ll}C_{\mu}, C_{1 \varepsilon}, & k-\varepsilon \text { model parameters: } 0.09,1.44,1.92 \\ C_{2 \varepsilon}, C_{3 \varepsilon} & \\ d_{p} & \text { particle nominal diameter, } \mathrm{m} \\ E_{1}, E_{2} & \text { Ergun's constants } \\ \vec{F}_{i} & \text { external body force } \\ \vec{g} & \text { gravitational acceleration, } 9.81 \mathrm{~m} / \mathrm{s}^{2} \\ G & \text { gas mass flux, } \mathrm{kg} / \mathrm{m}^{2} \mathrm{~s} \\ h_{i} & \text { specific enthalpy of the } i \text { th phase, } \mathrm{J} / \mathrm{kg} \\ \overline{\bar{I}} & \text { unitary shear stress tensor, } \mathrm{Pa} \\ k & k-\varepsilon \text { model kinetic energy } \\ L & \text { liquid mass flux, } \mathrm{kg} / \mathrm{m}^{2} \mathrm{~s} \\ p & \text { pressure, bar } \\ \vec{q}_{i} & \text { heat flux, } \mathrm{J} / \mathrm{m}^{2} \\ \vec{R}_{i} & \text { interphase momentum exchange term of } i \text { th } \\ & \text { phase } \\ R e_{i} & \text { Reynolds number of } i \text { th phase }\left[\left(\rho_{i} u_{i} d_{p} / \mu_{i}\right], \mathrm{di}-\right. \\ & \text { mensionless } \\ S_{q} & \text { source term of enthalpy due to chemical reaction } \\ t & \text { real time, min } \\ T & \text { temperature, } \mathrm{K} \\ \text { TOC } & \text { total organic carbon, } \mathrm{mg} / \mathrm{L} \\ \vec{u} & \text { superficialvector velocity, } \mathrm{m} / \mathrm{s}\end{array}$

\section{Greek letters}

$\begin{array}{ll}\alpha_{i} & \text { volume fraction of } i \text { th phase } \\ \varepsilon & k-\varepsilon \text { model dissipation energy } \\ \varepsilon_{G} & \text { gas holdup } \\ \varepsilon_{L} & \text { liquid holdup } \\ \varepsilon_{S} & \text { solid volume fraction } \\ \mu_{i} & \text { viscosity of } i \text { th phase, } \mathrm{Pa} \mathrm{s} \\ \rho_{i} & \text { density of } i \text { th phase, } \mathrm{kg} / \mathrm{m}^{3} \\ \Delta p & \text { total pressure drop, Pa } \\ \sigma_{k}, \sigma_{\varepsilon} & k-\varepsilon \text { model parameters: } 1.2,1.0 \\ \overline{\tau_{i}} & \text { shear stress tensor of } i \text { th phase, } \mathrm{Pa}\end{array}$

\section{Subscripts}

$\begin{array}{ll}G & \text { gas phase } \\ i & i \text { th phase } \\ L & \text { liquid phase } \\ S & \text { solid phase }\end{array}$

\section{Acknowledgement}

The Fundação para a Ciência e Tecnologia, Portugal, is gratefully acknowledged for the financial support.

\section{References}

Al-Dahhan, M.H., Larachi, F., Dudukovic, M.P., Laurent, A., 1997. High pressure trickle-bed reactors: a review. Industrial and Engineering Chemistry Research 36 (8), 3292-3314.

Attou, A., Ferschneider, G.A., 1999. Two-fluid model for flow regime transition in gas-liquid trickle-bed reactors. Chemical Engineering Science 54 (21), 5031-5037.

Bhargava, S.K., Tardio, J., Prasad, J., Foger, K., Akolekar, D.B., Grocott, S.C., 2006. Wet oxidation and catalytic wet oxidation. Industrial and Engineering Chemistry Research 45 (4), 1221-1258.

Carbonell, R.G., 2000. Multiphase flow models in packed beds. Oil \& Gas Science and Technology-Revue de 1'IFP 55, 417-442.

FLUENT 6.1, 2005. User's Manual to FLUENT 6.1. Fluent Inc., Centrera Resource Park, 10 Cavendish Court, Lebanon, USA.

GAMBIT 2, 2005. User's Manual to GAMBIT 2. Fluent Inc., Centrera Resource Park, 10 Cavendish Court, Lebanon, USA.

Gianetto, A., Specchia, V., 1992. Trickle-bed reactors: state of the art and perspectives. Chemical Engineering Science 47, 3197-3213.

Goto, S., Smith, J.M., 1975. Trickle bed reactors performance: i hold-up and mass transfer effects. A.I.Ch.E. Journal 21, 706-713.

Gunjal, P.R., Ranade, V.V., Chaudhari, R.V., 2005. Computational study of a single-phase flow in packed beds of spheres. A.I.Ch.E. Journal 51 (2), 365-378.

Holub, R.A., Dudukovic, M.P., Ramachandran, P.A., 1993. Pressure drop, liquid hold-up and flow regime transition in trickle flow. A.I.Ch.E. Journal 39 (2), 302-321.

Jiang, Y., Khadilkar, M.R., Al-Dahhan, M.H., Dudukovic, M.P., 2002. CFD modeling of multiphase in packed bed reactors: results and applications. A.I.Ch.E. Journal 48, 716-730.

Lopes, R.J.G., Silva, A.M.T., Quinta-Ferreira, R.M., 2007a. Screening of catalysts and effect of temperature for kinetic degradation studies of aromatic compounds during wet oxidation. Applied Catalysis B: Environmental 73 (1), 193-202.

Lopes, R.J.G., Silva, A.M.T., Quinta-Ferreira, R.M., 2007b. Kinetic modelling and trickle-bed CFD studies in the catalytic wet oxidation of vanillic acid. Industrial and Engineering Chemistry Research, http://dx.doi.org/10.1021/ ie070009a.

Nemec, D., Levec, J., 2005. Flow through packed bed reactors: 2. Two phase concurrent downflow. Chemical Engineering Science 60 (24), 6958-6970.

Paraskeva, P., Diamadopoulos, E., 2006. Technologies for olive mill wastewater (OMW) treatment: a review. Journal of Chemical Technology \& Biotechnology 81, 1475-1485.

Sáez, A.E., Carbonell, R.G., 1985. Hydrodynamic parameters for gas liquid concurrent flow in packed beds. A.I.Ch.E. Journal 31 (1), 52-62.

Sie, S.T., Krishna, R., 1998. Process development and scale up: III. Scale-up and scale-down of trickle bed processes. Reviews in Chemical Engineering 14, 203-252.

Taylor, R., Krishna, R., 1993. Multicomponent Mass Transfer. Wiley, New York.

van der Merwe, W., Nicol, W., 2005. Characterization of multiple flow morphologies within trickle flow regime. Industrial and Engineering Chemistry Research 44 (25), 9446-9450.

Wammes, W.J.A., Westerterp, K.R., 1990. The influence of reactor pressure on the hydrodynamics in a concurrent gas-liquid trickle-bed reactor. Chemical Engineering Science 45 (8), 2247-2254. 